\title{
LncRNA DANCR regulates lymphatic metastasis of bladder cancer via the miR-335/VEGF-C axis
}

\author{
Qinrong Ping ${ }^{\#}$, Yunqiang Shi”, Meng Yang, Hui Li, Yiming Zhong, Jian Li, Xiaofang Bi, Chunhui Wang^ \\ Department of Urology, Yan'an Hospital Affiliated to Kunming Medical University, Kunming, China \\ Contributions: (I) Conception and design: Q Ping, Y Shi; (II) Administrative support: Chunhui Wang; (III) Provision of study materials or patients: H \\ Li, Y Zhong; (IV) Collection and assembly of data: J Li, X Bi, M Yang; (V) Data analysis and interpretation: Q Ping, Y Shi, M Yang; (VI) Manuscript \\ writing: All authors; (VII) Final approval of manuscript: All authors. \\ \#These authors contributed equally to this work. \\ Correspondence to: Chunhui Wang. Department of Urology, Yan'an Hospital Affiliated to Kunming Medical University, 245 Renmin East Road, \\ Kunming 650000, China. Email: 13211604155@163.com.
}

Background: Substantial evidence indicate that long non-coding RNA (lncRNA) and microRNA (miRNA) act as key role in bladder cancer. Differentiation antagonistic ncRNA (DANCR) could be used as a biomarker in the occurrence and development of cancer. This study aims to explore the mechanism of DANCR/miR-335/VEGF-C axis affecting lymphatic metastasis of bladder cancer.

Methods: qRT-PCR detects the expression of DANCR in bladder cancer cell lines (SW780, 5637, T24, UM-UC-3) and normal bladder cell lines (SV-HUC-1), and selects T24 cell lines for subsequent experiments. The expression levels of DANCR, miR-335 and VEGF were measured by qRT-PCR, and the dual luciferase reporter gene verified the targeted regulation of DANCR on miR-335 and miR-335 on VEGF. CCK-8, Transwell and Wound healing assay detect the proliferation, invasion and migration ability of bladder cancer cells, Endothelial cell adhesion assay and Western blot further prove the lymphatic metastasis of bladder cancer.

Results: In this study, DANCR was highly expressed in bladder cancer cell lines. Transfection of siDANCR significantly inhibits the proliferation, migration, invasion and lymphatic metastasis of bladder cancer cells. Dual luciferase assay confirmed that DANCR targets miR-335/VEGF-C. Transfection of miR335 mimic promotes the proliferation, migration, invasion and lymphatic metastasis of bladder cancer cells, overexpression of DANCR eliminates the promotion of miR-335 mimic on bladder cancer cells. Further experiments proved that inhibition of miR-335 and overexpression of VEGF-C can reverse the inhibitory effect of silencing DANCR on bladder cancer cells.

Conclusions: In bladder cancer, DARCR plays an important role, which regulates the proliferation, migration, invasion and lymphatic metastasis of bladder cancer cells through the miR-335/VEGF-C molecular axis.

Keywords: Bladder cancer; DANCR; miR-335; VEGF-C; lymphatic metastasis

Submitted Dec 29, 2020. Accepted for publication Apr 17, 2021.

doi: $10.21037 /$ tau-21-226

View this article at: http://dx.doi.org/10.21037/tau-21-226

^ ORCID: Qinrong Ping, 0000-0003-2435-7760; Chunhui Wang, 0000-0002-6016-1768. 


\section{Introduction}

Bladder cancer (BC) is one of the most typical malignant tumors of the genitourinary system, and has high rates of recurrence rate and mortality (1-3). Although the treatment of $\mathrm{BC}$ has improved in the past few years, the 5 -year overall survival rate still shows a downward trend. Local or distant metastasis is the main cause of poor prognosis or death in patients with BC. The main metastases include lymphatic metastasis, hematogenous metastasis, direct diffusion, and implantation metastasis, of which lymphatic metastasis is the most common (4-6). At present, there is no early clinical diagnostic and interventional methods for BC lymphatic metastasis. The could be because the molecular mechanism of BC lymphatic metastasis has not been elucidated. Therefore, it is important to clarify the molecular mechanism of BC lymphatic metastasis.

Long non-coding RNA (lncRNA) is a new hot spot in the field of oncology research in recent years, and it is a type of ncRNA that does not have protein coding functions $(7,8)$. A large number of studies have shown that the abnormal expression of lncRNA in a variety of tumors is closely related to the occurrence and development of tumors (9-11). LncRNA can participate in the regulatory process of tumor cell growth, proliferation, invasion, and metastasis through the effects of oncogenes or tumor suppressor genes. In recent years, a large number of lncRNAs related to BC have been reported (12). For example, UBC1 is highly expressed in BC tissues and is closely related to patients' lymphatic metastasis and poor prognosis $(13,14)$. LncRNA BLACAT2 is significantly upregulated in $\mathrm{BC}$ and promotes related lymphangiogenesis and lymphatic metastasis (11). These suggested that lncRNA can be used as a therapeutic target for BC. Differentiation antagonistic non-protein coding RNA (DANCR) is highly expressed in ovarian cancer and promotes tumor growth and angiogenesis (12). More and more studies have shown that DANCR plays an important role in cancer progression. DANCR can also promote the metastasis and invasion of cervical cancer, pancreatic cancer, gastric cancer, and other cancer cells, which is related to poor prognosis of cancer (13-15). Zhan et al. found that DANCR promotes malignant phenotypes of bladder cancer cells by modulating the miR-149/MSI2 axis as a ceRNA (16). It suggests that DANCR may play an important role in bladder cancer. However, the mechanism of DANCR in lymphatic metastasis of BC needs further study.

The ceRNA hypothesis indicates that there is a regulatory network between lncRNA and microRNA
(miRNA). miRNAs are a group of small non-coding molecules with a length of about 18-25 nucleotides. Studies have found that miRNAs are involved in different stages of tumorigenesis and metastasis $(17,18)$. miR-335 is one of the miRNAs closely related to cancer progression, and may play a role in tumor suppression. Its expression is reduced in most human cancer cells (19-21). For example, miR-335 is significantly downregulated in gastric cancer and is considered to be a tumor suppressor for gastric cancer (22). However, the role of miR-335 in lymphatic metastasis of BC needs to be further explored.

Vascular endothelial growth factor (VEGF) is a specific heparin-binding growth factor for vascular endothelial cells that can induce the formation of new blood vessels (23). VEGF-C is a lymphangiogenic factor belonging to the VEGF family. Tumor cells can promote the formation of lymphatic vessels by expressing VEGF-C, leading to lymphatic metastasis of tumor cells (24-26). Studies have shown that VEGF-C is highly expressed in BC cells, while the expression level is low (or non-existent) in normal epithelial cells $(27,28)$. Detecting the expression level of VEGF-C in tissue can help predict the risk of pelvic lymphatic metastasis. Therefore, we propose that the DANCR/miR-335/VEGF-C axis plays a key role in lymphatic metastasis of $\mathrm{BC}$.

In the present study, we found that DANCR is highly expressed in BC patients and BC cell lines, and miR-335 is underexpressed. Using Cell Counting Kit-8 (CCK-8), Transwell, endothelial cell adhesion, and other assays confirmed that knockout of DANCR inhibited the proliferation, migration, and metastasis of $\mathrm{BC}$ cells, thereby regulating the process of lymphatic metastasis. In addition, we also revealed the regulatory mechanism of DANCR and miR-335 and of miR-335 and VEGF-C through dualluciferase reporter gene experiments. This suggests that DANCR can be used as an upstream regulatory factor to regulate the expression of miR-335 and VEGF-C, and affect tumor metastasis. The molecular axis of DANCR/ miR-335/VEGF-C plays an important role in lymphatic metastasis of $\mathrm{BC}$, increasing our understanding of the molecular mechanism of DANCR regulating lymphatic metastasis of BC. The aim of the presents study was to provide a new theoretical basis and experimental basis for the understanding of the mechanism of lymphatic metastasis of BC.

We present the following article in accordance with the MDAR reporting checklist (available at http://dx.doi. org/10.21037/tau-21-226). 


\section{Methods}

\section{Cell culture}

BC cells (5637, T24, UM-UC-3, and SW780 cell lines) and human normal bladder cell lines (SV-HUC-1) were purchased from the American Type Culture Collection (Manassas, VA, USA). The cells were cultured in RPMI 1640 medium (Thermo Fisher, Waltham, MA, USA), which contained $10 \%$ fetal bovine serum (Thermo Fisher, MA, USA), $100 \mathrm{IU} / \mathrm{mL}$ penicillin, and $100 \mu \mathrm{g} / \mathrm{mL}$ streptomycin (Invitrogen, Carlsbad, CA, USA). The cells were cultured at $37^{\circ} \mathrm{C}$ in a humidified incubator containing $5 \% \mathrm{CO}_{2}$.

\section{Quantitative reverse transcription polymerase chain reaction ( $q R T-P C R)$}

Using TRIzol reagent (Invitrogen, USA), total RNA was extracted from cells and tissue, and cDNA was synthesized by reverse transcription. SYBR Green (BioRad, Hercules, CA, USA) was used to detect the expression levels of DANCR, miR-335-5p, and VEGF-C; GAPDH and U6 were used as internal controls. The experiment was repeated 3 times, and the data were analyzed using the $2^{-\Delta \Delta \mathrm{Ct}}$ method. Primers were as follows: DANCR forward: 5'-GCCACAGGAGCTAGAGCAGT-3', reverse: 5'-GCAGAGTATTCAGGGTAAGGGT-3'; miR-335 forward: 5'-UGUUUUGAGCGGGGGUCAAGAGC-3', reverse: 5'-CUCUCAUUUGCUAUAUUCA-3'; VEGF-C forward: 5'-CACGAGCTACCTCAGCAAGA-3', reverse: 5 '-GCTGCCTGACACTGTGGTA-3'; GAPDH forward: 5'-GCAAGTTCAACGGCACAG-3', reverse: 5'-GCCAGTAGACTCCACGACAT-3'; and U6 forward: 5'-CTCGCTTCGGCAGCACATATACT-3', reverse: 5'-ACGCTTCACGAATTTGCGTGTC-3'.

\section{Western blot}

The total protein was extracted with RIPA lysis and extraction buffer (Thermo Fisher, Waltham, MA, USA), and the protein concentration was detected by bicinchoninic acid kit (Abcam, Cambridge, MA, USA). $10 \%$ sodium dodecylsulfate-polyacrylamide gel electrophoresis (SDS-PAGE) was selected to separate the protein according to the molecular weight of the target protein. After electrophoresis, the protein was transferred to a polyvinylidene difluoride membrane. After blocking with $5 \%$ skim milk powder at room temperature for $90 \mathrm{~min}$, the primary antibody was added at $4{ }^{\circ} \mathrm{C}$ overnight $(1: 1,000)$
(Abcam, Cambridge, MA, USA). The following day, the secondary antibody linked to horseradish peroxidase $(1: 1,000)$ (Abcam, Cambridge, MA, USA) was incubated at room temperature for $1 \mathrm{~h}$, and finally placed in ECL chemiluminescence solution (Beyotime, Shanghai, China) for imaging.

\section{CCK-8}

CCK-8 (Beyotime, Shanghai, China) was used to detect cell proliferation, Cells were grown in a $96-$ well plate at $37{ }^{\circ} \mathrm{C}$ for $24 \mathrm{~h}$, and then CCK- 8 solution was added and the cells were incubated for a further $1 \mathrm{~h}$. A microplate reader (Potenov, Beijing, China) was used to detect cell absorbance at a wavelength of $450 \mathrm{~nm}$, and the degree of cell growth was calculated.

\section{Transwell assay}

A Transwell migration chamber (Sigma Aldrich, St.Louis, MO, USA) was used to measure cell migration ability. The upper chamber was incubated with serum-free medium for $24 \mathrm{~h}$, and the lower chamber was incubated with RPMI 1640 medium containing $20 \%$ fetal bovine serum for $24 \mathrm{~h}$. The cells were then fixed with 4\% PFA for $10 \mathrm{~min}$ and stained with $0.1 \%$ crystal violet (Beyotime, Shanghai, China) for $30 \mathrm{~min}$.

\section{Wound healing}

Cells were placed in a 6-well plate and cultured overnight in normal cell growth medium. A $200-\mu \mathrm{m}$ sterile pipette was used to traverse the cell layer and incubate in another serum-free medium for $24 \mathrm{~h}$ prior to cell imaging.

\section{Endothelial cell adbesion}

BC cells were labeled with fluorescent dyes, and the excess dyes were rinsed off and add a small amount of serumfree medium. Endothelial cells were seeded onto a 96-well culture plate, and stained BC cells were added and cultured for $1 \mathrm{~h}$. Wash away the unadhered cells, the adhesion rate of bladder cancer cells and endothelial cells was detected using a confocal laser microscope (Olympus, Tokyo, Japan).

\section{Dual-luciferase reporter assay}

The wild and mutant plasmids of DANCR or VEGF-C 3' 


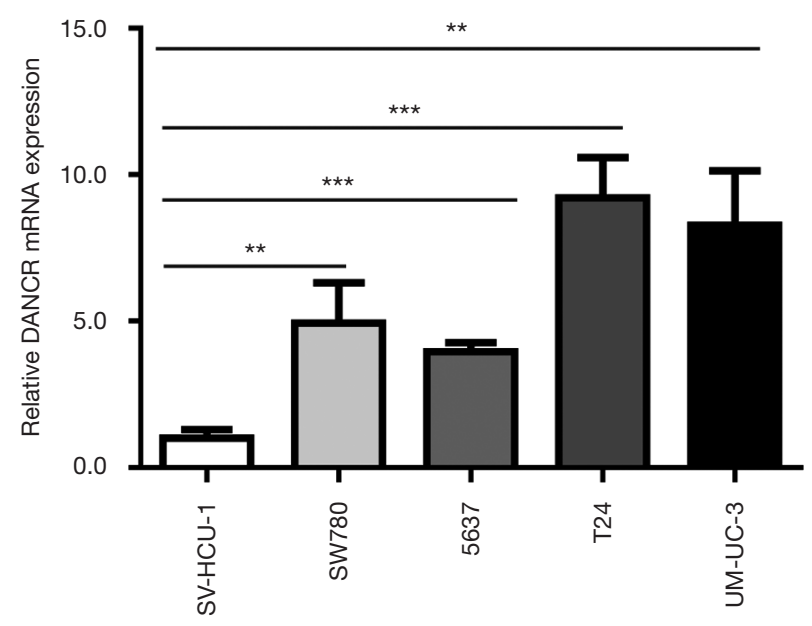

Figure 1 Differentiation antagonistic non-coding RNA (DANCR) was upregulated in bladder cancer cell lines. ${ }^{* *} \mathrm{P}<0.01$, ${ }^{* * *} \mathrm{P}<0.001$, compare to the SV-HUC-1 group.

untranslated region (UTR) containing miR-335-5p binding sites were constructed and integrated into a dual-luciferase vector, namely DANCR Wt/Mut or VEGF-C Wt/Mut. Subsequently, the plasmids were co-transfected with miR$335-5 \mathrm{p}$ mimics into 5,637 cells, and finally luciferase activity was detected with a dual-luciferase kit (Promega, Madison, WI, USA).

\section{Statistical analysis}

qRT-PCR, Western blot, and cell proliferation, migration, invasion, and other experiments were repeated 3 times. GraphPad Prism 5.0 software (GraphPad Software Inc., San Diego, CA, USA) was used for the statistical analysis, and all data were expressed as mean \pm standard deviation. Student's $t$-test was used for comparison between the 2 groups, $\mathrm{P}<0.05$ indicated statistical significance.

\section{Results}

\section{DANCR is bighly expressed in BC cell lines}

The qRT-PCR analysis showed that the expression level of DANCR was significantly higher in BC cell lines (SW780, 5637, T24, and UM-UC-3). Compared with the normal bladder cell line, SV-HUC-1, the expression of DANCR in BC cell lines T24 and UM-UC-3 increased significantly, and the expression in T24 was significantly more than that of UM-UC-3 (Figure 1). Therefore, in subsequent experiments, we will use the T24 cell line as a BC cell model.
Knockdown of DANCR inhibits lymphatic metastasis in BC

To study the biological function of DANCR in BC lymphatic metastasis, we silenced the expression of DANCR by transfecting DANCR interfering small RNA (siRNA) in the BC T24 cell line. We found that the transfection of DANCR siRNA can effectively inhibit the expression of DANCR, and that si-DANCR-3 had the best interference efficiency (Figure 2A). We chose si-DANC-3 for subsequent experiments to silence the expression of DANCR. We then used RTqPCR to detect the mRNA levels of miR-335 and VEGF-C, and si-NC was used as a control. After silencing DANCR (siDANCR), the expression of miR-335 was upregulated and the expression of VEGF-C was downregulated (Figure 2B). It was further verified by Western blot that, compared with the control group, the VEGF-C protein in the si-DANCR group was downregulated (Figure 2C). CCK-8 detection showed that the cell viability of the siDANCR group was significantly inhibited (Figure 2D). The Wound healing and Transwell assays also showed that si-DANCR significantly inhibited the migration and invasion rate of $\mathrm{BC}$ cells (Figure 2E,F). In addition, endothelial cell adhesion experiments further confirmed that the endothelial cell adhesion rate in the si-DANCR group was reduced (Figure 2G). Therefore, these results indicate that DANCR has a certain regulatory effect on lymphatic metastasis in BC.

\section{Targeting relationship of DANCR, VEGF-C, and miR-335}

To explore the interaction between DANCR, VEGF-C, and miR-335, we found that the miR-335 and DANCR, VEGF-C 3'-UTR sequence was highly matched using the StarBase Bioinformatics Network (Sun Yat-sen University, Guangdong, China) (Figure 3A,B). The dual-luciferase experiment also verified that miR-335 has targeted binding sites with DANCR and VEGF-C. We constructed DANCR and VEGF-C luciferase plasmids containing predicted sites or mutation sites (DANCR Wt/Mut, VEGF-C Wt/ Mut), miR-335 mimic or NC mimic is co-transfected with DANCR Wt/Mut or VEGF-C Wt/Mut to detect luciferase activity. The findings indicated that, co-transfection miR-335 mimic and DANCR/VEGF-C Wt significantly inhibited luciferase activity (Figure 3C,D).

\section{$D A N C R$ directly targets miR-335 to regulate lymphatic metastasis of $B C$}

To determine whether DANCR can regulate $\mathrm{BC}$ 


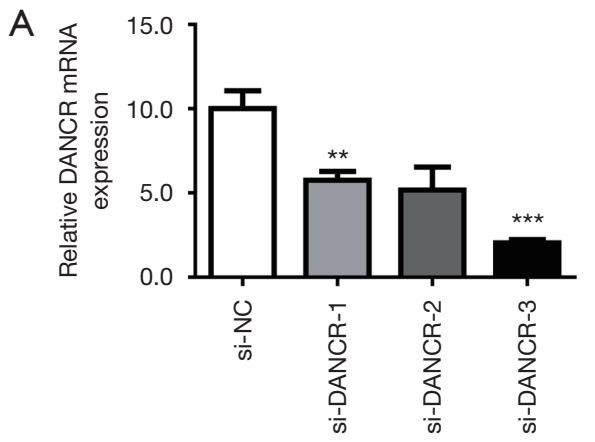

C

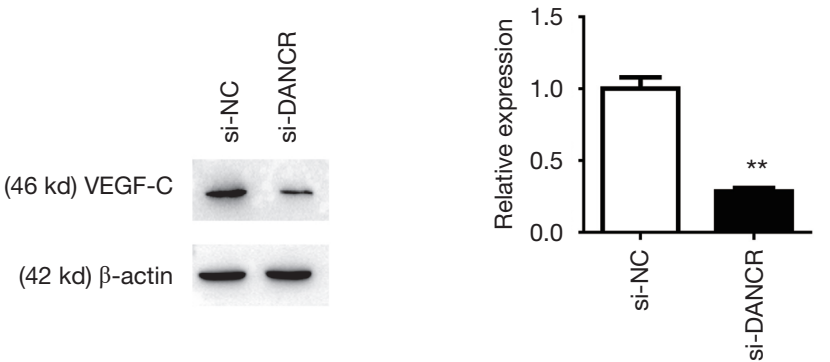

$\mathrm{E}$
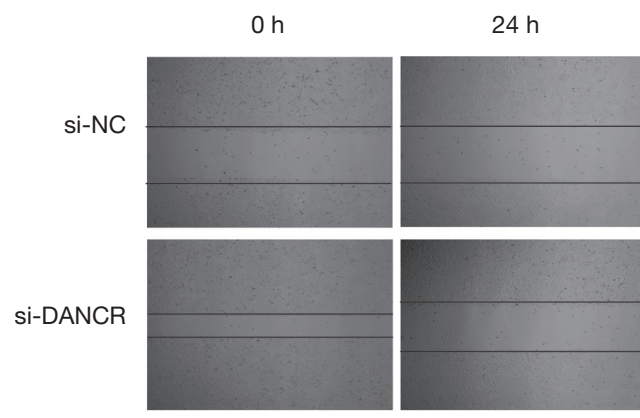

F

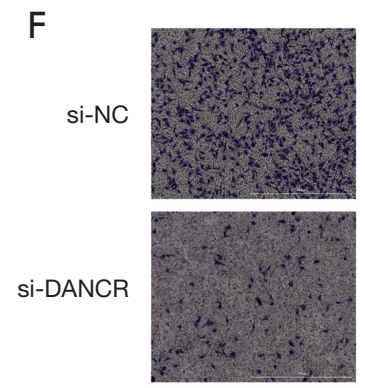

G

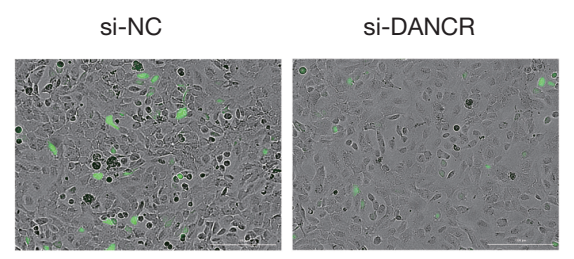

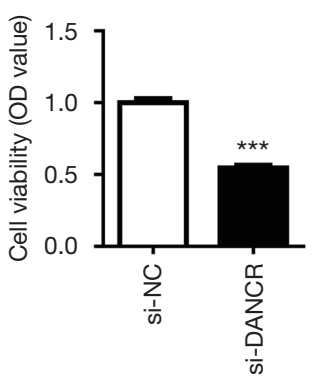

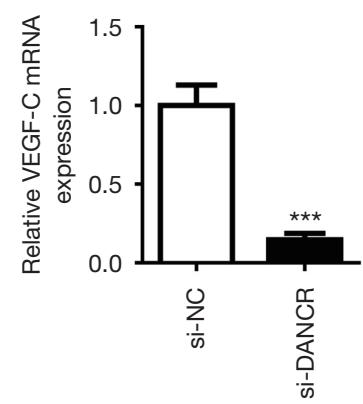

D

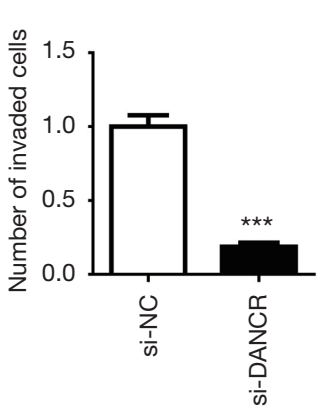

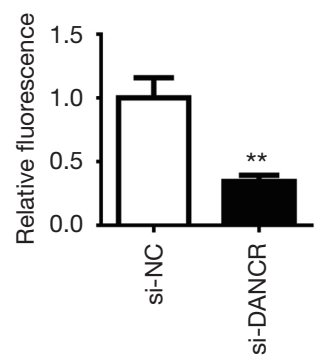

Figure 2 Differentiation antagonistic non-coding RNA (DANCR) regulated the proliferation, migration, invasion, and metastasis of bladder cancer cells. (A) Quantitative reverse transcription polymerase chain reaction (qRT-PCR) detection of DANCR siRNA transfection efficiency. ${ }^{* *} \mathrm{P}<0.01,{ }^{* * *} \mathrm{P}<0.001$ compare to the si-negative control (NC) group. (B) microRNA-355 (miR-335), vascular endothelial growth factor-C (VEGF-C) mRNA expression level using qRT-PCR. ${ }^{* *} \mathrm{P}<0.01,{ }^{* * *} \mathrm{P}<0.001$ compare to the si-NC group (C) VEGF-C expression level using Western blot ${ }^{* *} \mathrm{P}<0.01$ compare to the si-NC group. (D,E,F) si-DANCR inhibited the proliferation, migration, and invasion of bladder cancer cells, as observed by Cell Counting Kit-8, Wound healing, and Transwell assay (Crystal violet staining, Scale bar =1,000 $\mu \mathrm{m}$, ${ }^{* * *} \mathrm{P}<0.001$ compare to the si-NC group). (G) Knockout DANCR reduced bladder cancer metastasis through endothelial cell adhesion assay. (CFDA-SE staining, Scale bar $=200 \mu \mathrm{m},{ }^{* *} \mathrm{P}<0.01$ compare to the si-NC group). OD, optical density. 


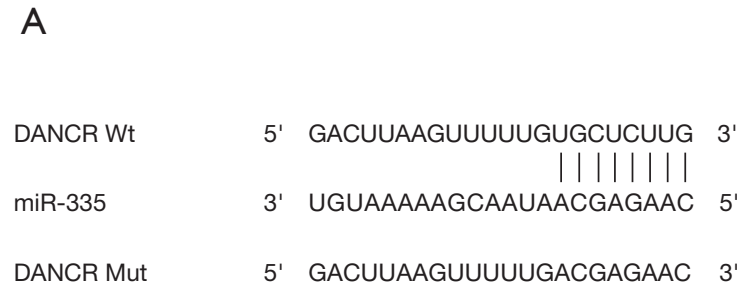

B

$\begin{array}{lll}\text { VEGF-C Wt } & 5^{\prime} & \text { AACAGCCAAGAUUUUCCUCUUGU } \\ \text { miR-335 } & 3^{\prime} & \text { UGUAAAAAGCAAUAACGAGAACU } \\ \text { VEGF-C Mut } & 5^{\prime} & \text { AACAGCCAAGAUUAACGAGAACU }\end{array}$

C

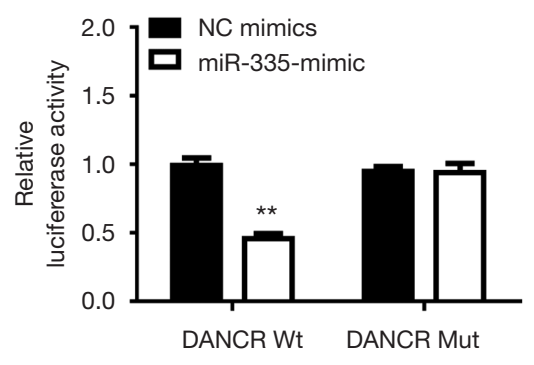

D

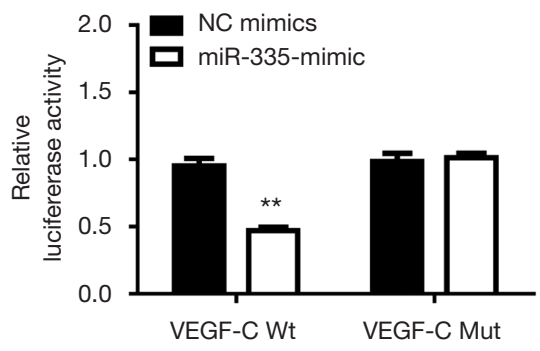

Figure 3 Targeting between differentiation antagonistic non-coding RNA (DANCR), vascular endothelial growth factor-C (VEGF-C), and microRNA-335 (miR-335). (A) Binding site of miR-335 and DANCR was predicted by StarBase. (B) Binding site of miR-335 and VEGF-C was predicted by StarBase. (C) Dual-luciferase report assay demonstrated that co-transfection DANCR Wild type (Wt) and miR-335 mimic significantly inhibited luciferase activity. (D) Dual-luciferase report assay demonstrated that co-transfected VEGF-C Wt and miR-335 mimic significantly inhibited luciferase activity. ${ }^{*} \mathrm{P}<0.01$ compare to the co-transfection DANCR/VEGF-C Wt and Negative control (NC) mimic group. DANCR/VEGF-C Mutation (Mut).

lymphatic metastasis by combining with miR-335, the mRNA expression of miR-335 and VEGF-C after the transfection of miR-335 mimic was detected by qRT-PCR. Compared with the control group, the expression of miR335 increased, and the overexpression of DANCR reduced this change (Figure 4A). The qRT-PCR and Western blot results showed that miR-335 mimic can significantly reduce the expression of VEGF-C in the T24 cell line; however, the overexpression of DANCR can reverse this change (Figure 4A,B). CCK-8, Wound healing, and Transwell assays showed that the miR-335 mimic group inhibited the vitality, migration, and invasion ability of $\mathrm{BC}$ cells, and the overexpression of DANCR released the inhibition (Figure $4 C, D, E$ ). Similarly, the results of the endothelial cell adhesion experiments showed that the endothelial cell adhesion rate decreased after the miR-335 mimic was added, and DANCR overexpression alleviated the decreased endothelial cell adhesion rate (Figure 4F). These data suggest that DANCR targets miR-335 and affects BC lymphatic metastasis.

\section{Inhibition of miR-335 or overexpression of VEGF-C reverse the effect of si-DANCR}

To understand the influence of miR-335 or VEGF-C expression on the DANCR regulatory mechanism in BC lymphatic metastasis, we constructed a recovery experiment. Compared with the control group, qRT-PCR found that the expression of DANCR was downregulated after si-DANCR transfection, and the expression of miR-335 was upregulated. The inhibition of miR-335 or the overexpression of VEGF-C reversed this change (Figure 5A). qRT-PCR and Western blot showed that the expression of VEGF-C in the si-DANCR group was significantly downregulated, after inhibiting miR-335 or overexpressing VEGF-C, the expression of VEGF-C increases, which is not significantly different from the control group (Figure $5 A, B$ ). The CCK-8 results showed that the downregulation of miR-335 or the upregulation of VEGF-C reversed the inhibition of si-DANCR cell viability (Figure 5C). In addition, the Wound healing and Transwell 
A

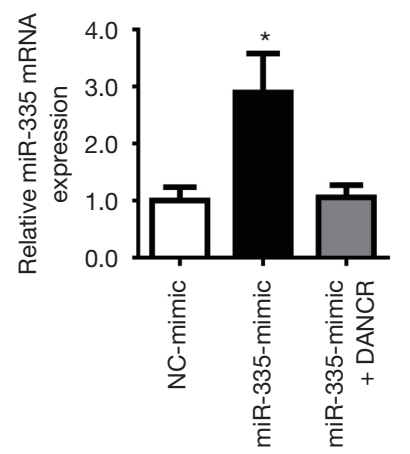

C

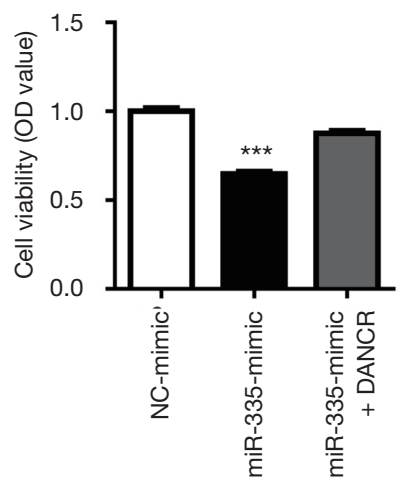

$\mathrm{E}$

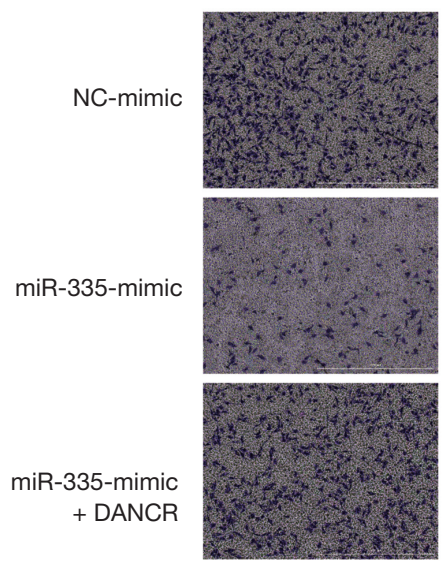

B

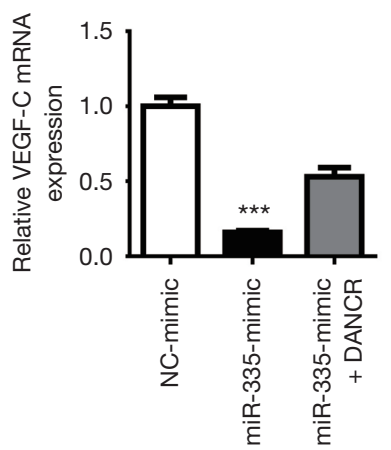

D

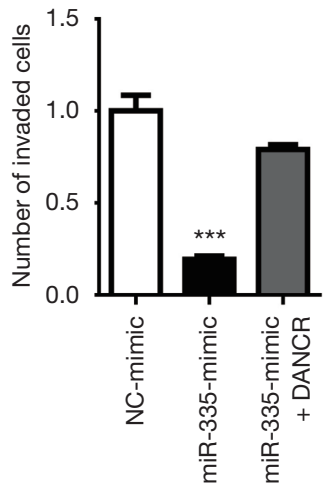

$0 \mathrm{~h}$

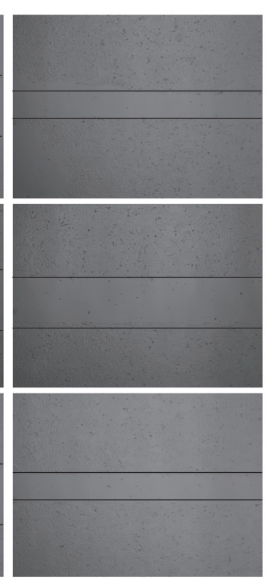

$\mathrm{F}$

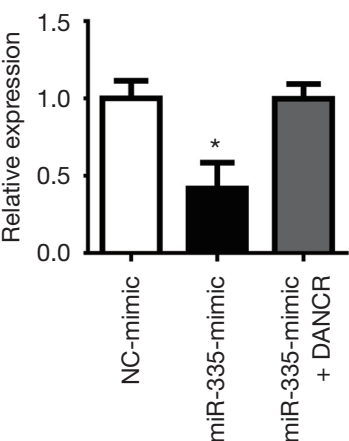

$24 \mathrm{~h}$

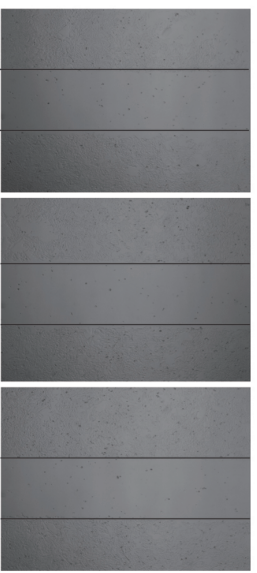

NC-mimic

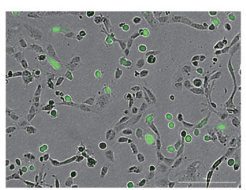

miR-335-mimic

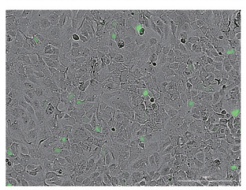

+ DANCR

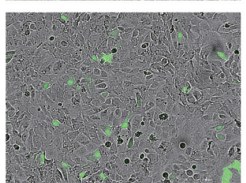

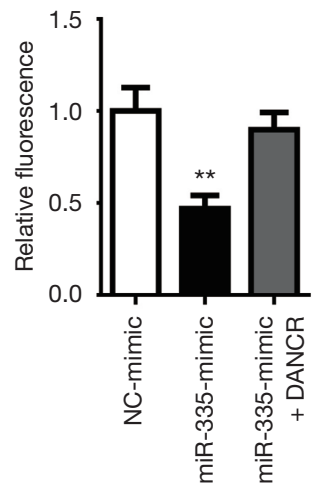

miR-335-mimic

Figure 4 Differentiation antagonistic non-coding RNA (DANCR) regulated lymphatic metastasis via microRNA-355 (miR-335). (A) Quantitative reverse transcription polymerase chain reaction detected mRNA expression levels of miR-335 and vascular endothelial growth factor-C (VEGF-C) after miR-335 overexpression. ${ }^{*} \mathrm{P}<0.05$, ${ }^{* * *} \mathrm{P}<0.001$, compare to Negative Control (NC) mimic group. (B) Western blot determined the expression level of VEGF-C. ${ }^{*}<<0.05$ compare to NC mimic group. (C) Cell Counting Kit- 8 results showed that miR335 increased the inhibition of bladder cancer cell proliferation. ${ }^{* *} \mathrm{P}<0.001$ compare to NC mimic group. (D-F) miR-335 mimic inhibited cell migration, invasion (Crystal violet staining, Scale bar $=1,000 \mu \mathrm{m},{ }^{* * *} \mathrm{P}<0.001$ compare to NC mimic group), and metastasis (CFDA-SE staining, Scale bar $=200 \mu \mathrm{m},{ }^{* *} \mathrm{P}<0.01$ compare to NC mimic group) through Wound healing, Transwell, and endothelial cell adhesion assay. OD, optical density. 

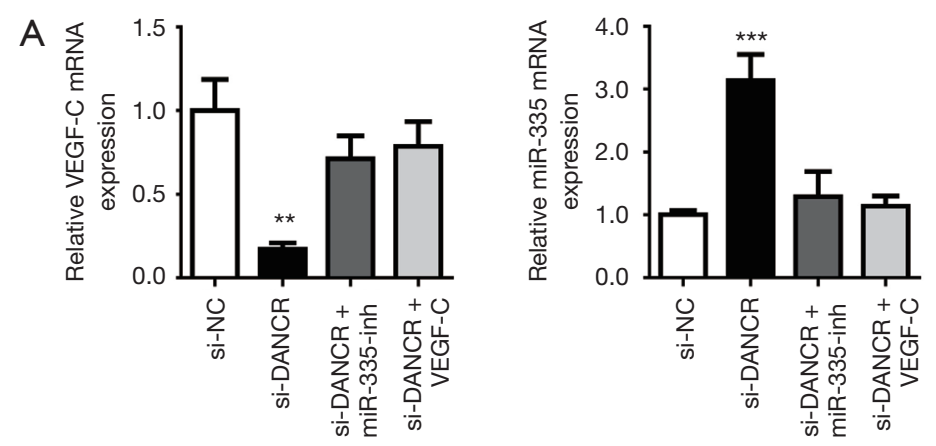

B
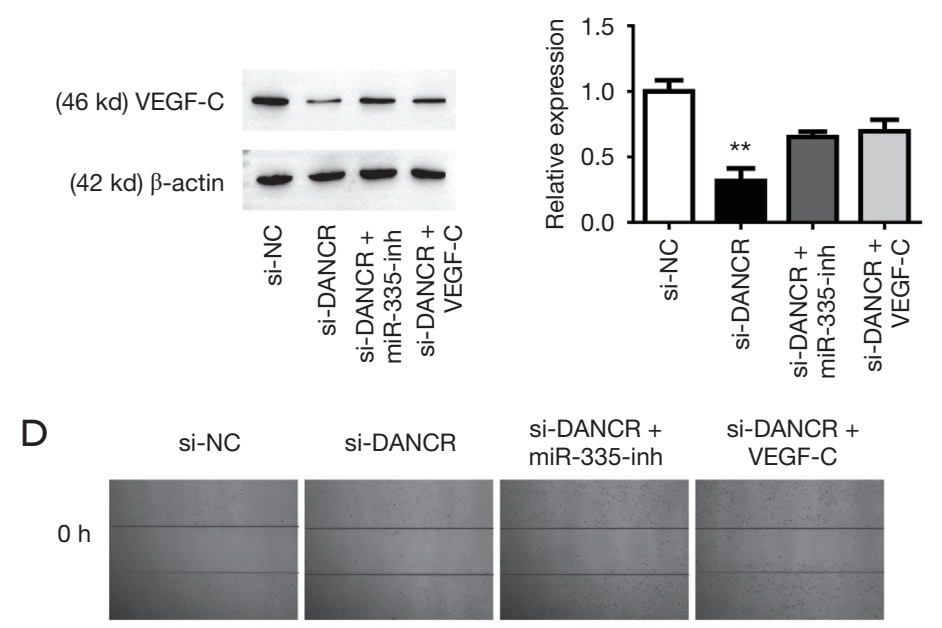

Si-DANCR

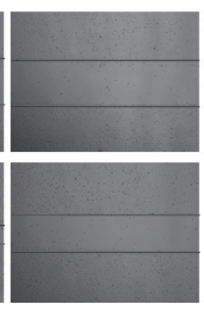

F

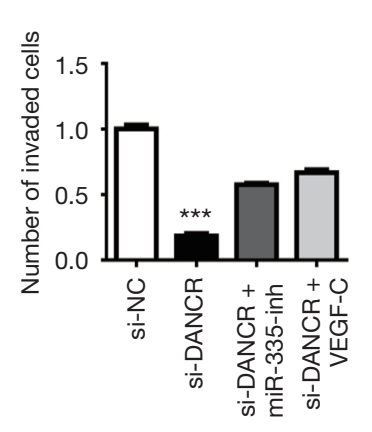
$\begin{array}{lc}\text { Si-DANCR }+ & \text { Si-DANCR }+ \\ \text { miR-335-inh } & \text { VEGF-C }\end{array}$
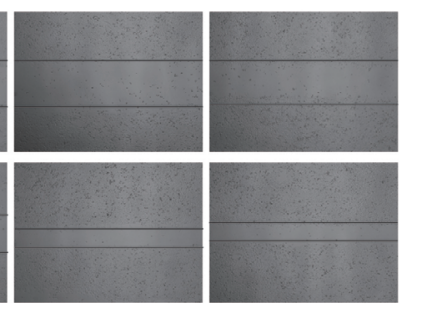

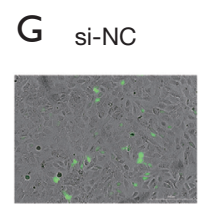

si-DANCR

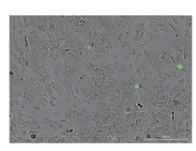

Si-DANCR + miR-335-inh

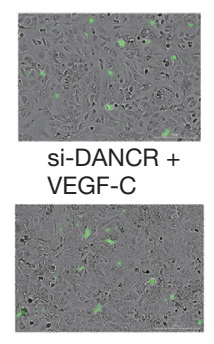

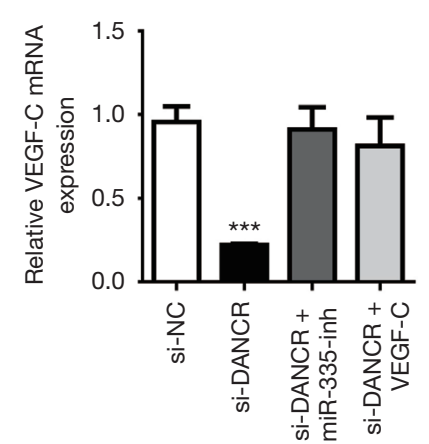

C

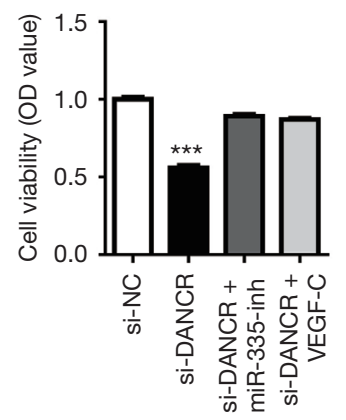

E
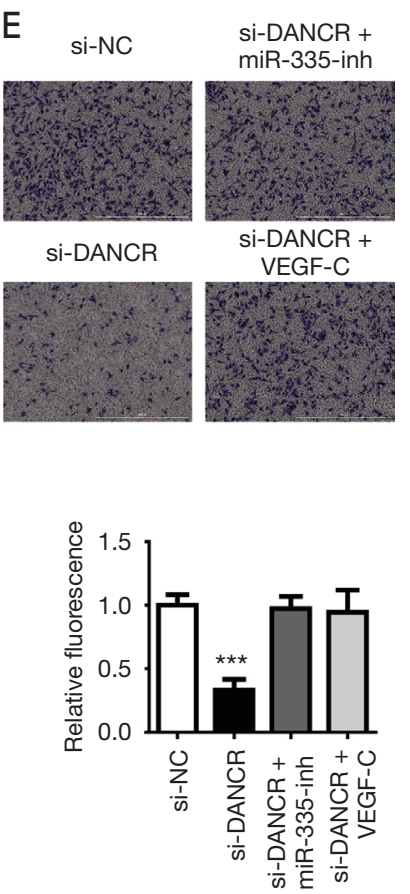

Figure 5 Differentiation antagonistic non-coding RNA (DANCR)/microRNA-355 (miR-335)/vascular endothelial growth factor-C (VEGF-C) axis regulated tumor characteristics of bladder cancer cells. (A) Quantitative reverse transcription polymerase chain reaction revealed the expression levels of DANCR, miR-335, and VEGF-C. ${ }^{*} \mathrm{P}<0.01,{ }^{* *} \mathrm{P}<0.001$ compare to the si-Negative Control (NC). (B) Western blot detection of VEGF-C expression after si-DNACR. ${ }^{* *} \mathrm{P}<0.01$ compare to the si-NC. (C-G) Inhibition of miR-335 or overexpression of VEGF-C after knockout DANCR restored the proliferation ${ }^{* * *} \mathrm{P}<0.001$ compare to the si-NC), migration, invasion (Crystal violet staining, Scale bar $=1,000 \mu \mathrm{m},{ }^{* * *} \mathrm{P}<0.001$ compare to the si-NC), and metastasis (CFDA-SE staining, Scale bar $=200 \mu \mathrm{m}$, *** $\mathrm{P}<0.001$ compare to the si-NC) of bladder cancer cells. OD, optical density. 
assays found that the cell migration and invasion of siDANCR was significantly lower than that of the control group, while inhibition of miR-335 or overexpression of VEGF-C made up for this inhibitory effect on cell migration and invasion (Figure $5 D, E, F$ ). In addition, cell endothelial adhesion experiments further verified these findings (Figure $5 G$ ). The downregulation of miR-335 or upregulation of VEGF-C can reverse the inhibition of DANCR deletion in lymphatic metastasis of BC. VEGF-C is essential for lymphatic metastasis. These results indicate that the DANCR/miR-335/VEGF-C molecular axis regulates the occurrence and development of $\mathrm{BC}$ lymphatic metastasis.

\section{Discussion}

$\mathrm{BC}$ is one of the most common malignant tumors in the urinary system. It has the characteristics of painless gross hematuria, dysuria or urinary retention, which seriously threatens human life and health. The current treatments for $\mathrm{BC}$ include chemotherapy, surgery, and radiation therapy; however, patients still have recurrence or poor prognosis (29-31), mainly characterized by lymphatic metastasis. The lack of a comprehensive understanding of the occurrence and development of $\mathrm{BC}$ lymphatic metastasis is one of the important reasons for the decline in patient survival.

In recent years, it has been found that lncRNAs plays an important role in the regulation of gene expression in BC. For example, UCA1 and LNMAT1 may be used as therapeutic targets for patients with $\mathrm{BC}$, and the abnormal expression of lncRNA is associated with its occurrence $(32,33)$. These indicate that IncRNA can be used as an oncogene or as a tumor suppressor gene. As a newly discovered lncRNA, DANCR is abnormally expressed in a variety of human cancers. For example, Qu et al. reported that DANCR is upregulated in oral squamous cell carcinoma tissues and cells. Si-DANCR can reduce the proliferation, migration, and invasion of OSCC cells through miR-216a (34). Pan et al. also found that DANCR can be activated by SALL4 and promote the proliferation and invasion of gastric cancer cells (35). However, the specific downstream targeting molecule of DANCR in $\mathrm{BC}$ remains to be explored. Our research has found that DANCR is highly expressed in BC cell lines. The downregulation of DANCR has an inhibitory effect on the vitality, migration, and invasion of $\mathrm{BC}$ cells. In addition, Western blot and endothelial cell adhesion assay confirmed that DANCR affects lymphatic metastasis. These results indicate that DANCR plays an important role in the development of BC lymphatic metastasis.

Studies have shown that miRNAs may play an indispensable role in cancer cell proliferation, differentiation, invasion, and migration, and many miRNA expression disorders are related to lymphatic metastasis of various cancers (36-38). For example, Guo et al. found that miR-34a has a tumor suppressor effect in mouse liver cancer, not only inhibiting cell growth but also inhibiting metastasis, and can be used as a new therapeutic target for liver cancer (39). In addition, there is a regulatory relationship between lncRNA and miRNA; IncRNA can affect the downstream target genes of miRNA by regulating miRNA, thereby affecting the occurrence and development of various cancers $(40,41)$. In the present study, we used dual-luciferase report experiments to verify the targeting relationship between miR-335 and DANCR, adding miR-335 mimic to inhibit the migration, invasion, and metastasis of $\mathrm{BC}$ cells; the overexpression of DANCR reversed this inhibition. Therefore, miR-335 may be a target of DANCR in BC, and DANCR promotes lymphatic metastasis of $\mathrm{BC}$ by inhibiting miR-335.

VEGF-C was the first lymphangiogenic factor discovered that affects tumor blood vessel formation and cancer progression. We found that the expression of VEGF-C was upregulated in $\mathrm{BC}$ cells. Luciferase experiments further verified the targeting relationship between miR-335 and VEGF-C. The si-DANCR group not only inhibited the vitality, migration, and invasion of $\mathrm{BC}$ cells but also inhibited metastasis, which was counteracted by inhibiting miR-335 or overexpressing VEGF-C. Therefore, we believe that the downregulation of DANCR inhibits the expression of VEGF-C through the upregulation of miR-335, and the molecular axis of DANCR/miR-335/NEGF-C can be used as a target to explore lymphatic metastasis of BC.

In summary, we found that DANCR promotes lymphatic metastasis of $\mathrm{BC}$, indicating that DANCR targets the molecular mechanism of miR-335 in lymphatic metastasis of BC and affects VEGF-C expression, and determines the molecular mechanism of the DANCR/ miR-335/VEGF-C axis. The existence of the axis could be used to discover potential markers of $\mathrm{BC}$ lymphatic metastasis and targets for the diagnosis and treatment of $\mathrm{BC}$.

\section{Acknowledgments}

Funding: This article was supported by grants from the Basic Research Project of Yunnan Provincial: Kunming Medical 
University Joint Special Project (Grant No. 2018FE001090), Medical Discipline Leader Project of Yunnan Provincial (Grant No. D-2017044), Open Project from the Tumor Immunization and Prevention Key Experiment Laboratory of Yunnan Provincial (Grant No. 2017DG00404), Scientific Research Fund from the Education Department of Yunnan Provincial (Grant No. 2020J0218), Health Science and Technology Talent Training Project of Kunming City (Grant No. 2020-SW-12), and Health Research Project from the Kunming Municipal Health Commission (Grant No. 2020-04-05-011).

\section{Footnote}

Reporting Checklist: The authors have completed the MDAR reporting checklist. Available at http://dx.doi.org/10.21037/ tau-21-226

Data Sharing Statement: Available at http://dx.doi. org/10.21037/tau-21-226

Conflicts of Interest: All authors have completed the ICMJE uniform disclosure form (available at http://dx.doi. org/10.21037/tau-21-226). The authors have no conflicts of interest to declare.

Ethical Statement: The authors are accountable for all aspects of the work in ensuring that questions related to the accuracy or integrity of any part of the work are appropriately investigated and resolved.

Open Access Statement: This is an Open Access article distributed in accordance with the Creative Commons Attribution-NonCommercial-NoDerivs 4.0 International License (CC BY-NC-ND 4.0), which permits the noncommercial replication and distribution of the article with the strict proviso that no changes or edits are made and the original work is properly cited (including links to both the formal publication through the relevant DOI and the license). See: https://creativecommons.org/licenses/by-nc-nd/4.0/.

\section{References}

1. Antoni S, Ferlay J, Soerjomataram I, et al. Bladder Cancer Incidence and Mortality: A Global Overview and Recent Trends. Eur Urol 2017;71:96-108.

2. Skeldon SC, Larry Goldenberg S. Bladder cancer: a portal into men's health. Urol Oncol 2015;33:40-44.
3. Kang Z, Li Y, Yu Y, et al. Research progress on bladder cancer molecular genetics. J Cancer Res Ther 2014;10 Suppl:C89-94.

4. Xie R, Chen X, Chen Z, et al. Polypyrimidine tract binding protein 1 promotes lymphatic metastasis and proliferation of bladder cancer via alternative splicing of MEIS2 and PKM. Cancer Lett 2019;449:31-44.

5. Seiler R, Lam LL, Erho N, et al. Prediction of Lymph Node Metastasis in Patients with Bladder Cancer Using Whole Transcriptome Gene Expression Signatures. J Urol 2016;196:1036-41.

6. Liedberg F, Månsson W. Lymph node metastasis in bladder cancer. Eur Urol 2006;49:13-21.

7. Peng WX, Koirala P, Mo YY. LncRNA-mediated regulation of cell signaling in cancer. Oncogene 2017;36:5661-7.

8. Li J, Meng H, Bai Y, et al. Regulation of lncRNA and Its Role in Cancer Metastasis. Oncol Res 2016;23:205-17.

9. He W, Cai Q, Sun F, et al. linc-UBC1 physically associates with polycomb repressive complex 2 (PRC2) and acts as a negative prognostic factor for lymph node metastasis and survival in bladder cancer. Biochim Biophys Acta 2013;1832:1528-37.

10. Zhang S, Du L, Wang L, et al. Evaluation of serum exosomal LncRNA-based biomarker panel for diagnosis and recurrence prediction of bladder cancer. J Cell Mol Med 2019;23:1396-405.

11. He W, Zhong G, Jiang N, et al. Long noncoding RNA BLACAT2 promotes bladder cancer-associated lymphangiogenesis and lymphatic metastasis. J Clin Invest 2018;128:861-75.

12. Lin X, Yang F, Qi X, et al. LncRNA DANCR promotes tumor growth and angiogenesis in ovarian cancer through direct targeting of miR-145. Mol Carcinog 2019;58:2286-96.

13. Luo Y, Wang Q, Teng L, et al. LncRNA DANCR promotes proliferation and metastasis in pancreatic cancer by regulating miRNA-33b. FEBS Open Bio 2020;10:18-27.

14. Mao Z, Li H, Du B, et al. LncRNA DANCR promotes migration and invasion through suppression of lncRNA-LET in gastric cancer cells. Biosci Rep 2017;37:BSR20171070.

15. Liang H, Zhang C, Guan H, et al. LncRNA DANCR promotes cervical cancer progression by upregulating ROCK1 via sponging miR-335-5p. J Cell Physiol 2019;234:7266-78.

16. Zhan Y, Chen Z, Li Y, et al. Long non-coding RNA DANCR promotes malignant phenotypes of bladder 
cancer cells by modulating the miR-149/MSI2 axis as a ceRNA. J Exp Clin Cancer Res 2018;37:273.

17. Lee YS, Dutta A. MicroRNAs in cancer. Annu Rev Pathol 2009;4:199-227.

18. Rupaimoole R, Slack FJ. MicroRNA therapeutics: towards a new era for the management of cancer and other diseases. Nat Rev Drug Discov 2017;16:203-22.

19. Gao Y, Zeng F, Wu JY, et al. MiR-335 inhibits migration of breast cancer cells through targeting oncoprotein c-Met. Tumour Biol 2015;36:2875-83.

20. Luo LJ, Wang DD, Wang J, et al. Diverse roles of miR335 in development and progression of cancers. Tumour Biol 2016;37:1-12

21. Zhang P, Yang X, Wang L, et al. Overexpressing miR-335 inhibits DU145 cell proliferation by targeting early growth response 3 in prostate cancer. Int J Oncol 2019;54:1981-94.

22. Sandoval-Bórquez A, Polakovicova I, Carrasco-Véliz $\mathrm{N}$, et al. MicroRNA-335-5p is a potential suppressor of metastasis and invasion in gastric cancer. Clin Epigenetics 2017;9:114.

23. Siveen KS, Prabhu K, Krishnankutty R, et al. Vascular Endothelial Growth Factor (VEGF) Signaling in Tumour Vascularization: Potential and Challenges. Curr Vasc Pharmacol 2017;15:339-51.

24. Chaudary N, Milosevic M, Hill RP. Suppression of vascular endothelial growth factor receptor 3 (VEGFR3) and vascular endothelial growth factor C (VEGFC) inhibits hypoxia-induced lymph node metastases in cervix cancer. Gynecol Oncol 2011;123:393-400.

25. Wang J, Huang Y, Zhang J, et al. Pathway-related molecules of VEGFC/D-VEGFR3/NRP2 axis in tumor lymphangiogenesis and lymphatic metastasis. Clin Chim Acta 2016;461:165-71.

26. Lim L, Bui H, Farrelly O, et al. Hemostasis stimulates lymphangiogenesis through release and activation of VEGFC. Blood 2019;134:1764-75.

27. Zu X, Tang Z, Li Y, et al. Vascular endothelial growth factor-C expression in bladder transitional cell cancer and its relationship to lymph node metastasis. BJU Int 2006;98:1090-93.

28. Zhou XU, Qi L, Tong S, et al. miR-128 downregulation promotes growth and metastasis of bladder cancer cells and involves VEGF-C upregulation. Oncol Lett 2015;10:3183-90.

29. Zhang H, Jiang M, Liu Q, et al. miR-145-5p inhibits the proliferation and migration of bladder cancer cells by targeting TAGLN2. Oncol Lett 2018;16:6355-60.

30. Wang J, Zhang H, Situ J, et al. KCNQ1OT1 aggravates cell proliferation and migration in bladder cancer through modulating miR-145-5p/PCBP2 axis. Cancer Cell Int 2019;19:325.

31. Crabb SJ, Douglas J. The latest treatment options for bladder cancer. Br Med Bull 2018;128:85-95.

32. Li HJ, Sun XM, Li ZK, et al. LncRNA UCA1 Promotes Mitochondrial Function of Bladder Cancer via the MiR195/ARL2 Signaling Pathway. Cell Physiol Biochem 2017;43:2548-61.

33. Chen $\mathrm{C}$, He W, Huang J, et al. LNMAT1 promotes lymphatic metastasis of bladder cancer via CCL2 dependent macrophage recruitment. Nat Commun 2018;9:3826.

34. Qu XH, Shi YL, Ma Y, et al. LncRNA DANCR regulates the growth and metastasis of oral squamous cell carcinoma cells via altering miR-216a-5p expression. Hum Cell 2020;33:1281-93.

35. Pan L, Liang $W, \mathrm{Gu} J$, et al. Long noncoding RNA DANCR is activated by SALL4 and promotes the proliferation and invasion of gastric cancer cells. Oncotarget 2017;9:1915-30.

36. Wu L, Hu B, Zhao B, et al. Circulating microRNA-422a is associated with lymphatic metastasis in lung cancer. Oncotarget 2017;8:42173-88.

37. Zhang WW, Ming XL, Rong Y, et al. Diagnostic Value Investigation and Bioinformatics Analysis of miR-31 in Patients with Lymph Node Metastasis of Colorectal Cancer. Anal Cell Pathol (Amst) 2019;2019:9740475.

38. Huang KH, Lan YT, Fang WL, et al. The correlation between miRNA and lymph node metastasis in gastric cancer. Biomed Res Int 2015;2015:543163.

39. Guo Y, Li S, Qu J, et al. MiR-34a inhibits lymphatic metastasis potential of mouse hepatoma cells. Mol Cell Biochem 2011;354:275-282.

40. Guo L, Zhao Y, Yang S, et al. An integrated analysis of miRNA, lncRNA, and mRNA expression profiles. Biomed Res Int 2014;2014:345605.

41. Paraskevopoulou MD, Hatzigeorgiou AG. Analyzing MiRNA-LncRNA Interactions. Methods Mol Biol 2016;1402:271-86.

Cite this article as: Ping Q, Shi Y, Yang M, Li H, Zhong Y, Li J, Bi X, Wang C. LncRNA DANCR regulates lymphatic metastasis of bladder cancer via the miR-335/VEGF-C axis. Transl Androl Urol 2021;10(4):1743-1753. doi: 10.21037/tau$21-226$ 